\title{
Características de las adolescentes obstétricas Hospital Universitario de Caldas
}

\author{
Carlos Alberto Esparza Duque*; Mario Calle Mesa**
}

RESUMEN: Se hace un estudio descriptivo, prospectivo, de 576 adolescentes embarazadas, atendidas en el Servicio de Obstetricia del Hospital Universitario de Caldas, entre el 1o. de enero y el 31 de diciembre de 1989.

Se intenta comparar nuestros resultados con algunos publicados en la literatura nacional de la especialidad.

La edad, talla y peso promedio fueron 16.9 años, $59.5 \mathrm{~kg}$., y $155 \mathrm{~cm}$., respectivamente.

El $85,1 \%$ eran primigestantes. En cuanto a morbilidad se encontró un $7.8 \%$ de preeclampsia-eclampsia. El $77.1 \%$ de los partos fueron normales. $22.9 \%$ intervenidos o en presentación de pelvis.

La incidencia de cesáreas fue de $\mathbf{1 5 . 8 \%}$. De éstas, el $\mathbf{1 6 . 4 \%}$ se debieron a presentación de pelvis en primigestantes. Los partos en esta presentación se atienden por cesárea en un $80.8 \%$.

La comparación con otros estudios es muy difícil, debido a la gran heterogeneidad de los mismos. Algunos de los resultados de este intento, quedan consignados en la Tabla 25.

En un próximo estudio compararemos el grupo de adolescentes primigestantes con otro de primigestantes de 19 a 30 años, con el fin de analizar si existen o no diferencias significativas entre ambos.

PALABRAS CLAVES: adolescente, embarazo

SUMMARY: 576 pregnant adolescents were prospectively studied at the Obstetrical Service of the Caldas University Hospital, from january 1st to december 31st 1989.

We tried to compare our results with some others published in national specialized literature.

Mean values for age, height and weight were 16.9 years, $59.5 \mathrm{~kg}$. and $155 \mathrm{~cm}$. respectively.

$85.1 \%$ were primigravid adolescents. Preeclampsia-eclampsia was diagnosed in $7.8 \%$ of cases. $77.1 \%$ of deliveries were normal and $22.9 \%$ operative or breech deliveries.

Incidence of cesarean section was $15.8 \% .16 .4 \%$ of them were done because of breech presentation in primigravid adolescents. $80.8 \%$ of breech deliveries are managed trough cesarean section.

Comparison of our results with those of other investigators was very difficult because of the great heterogeneity of study designs. Some of the results are shown in Table 25 .

In the second part of this study we are going to compare the group of primigravid primiparous adolescents with primigravid primiparous women 19 to 30 years old in order to analize if there are significant differences between them.

KEY WORDS: adolescent, pregnancy

\section{Introducción}

Revisando la literatura nacional gineco-obstétrica, sobre problemas de adolescentes, encontramos 25 trabajos publicados entre los años de 1972 y 1990 , es decir un promedio de 1.4 por año.

* Profesor Titular, Facultad de Medicina. Universidad de Caldas.

** Profesor Titular, Facultad de Medicina. Universidad de Caldas.
El contenido de los mismos es muy heterogéneo. No hay acuerdo ni siquiera en cuanto a los límites etáreos de la adolescencia. Algunos sólo estudian mujeres de 13 a 15 años (6). Otros incluyen las personas de 11 a 17 años $(1,3)$.

El número de mujeres adolescentes en Colombia se estima en 3'000.000 y con una tasa de fecundidad natural de 74.0 por mil, se esperarían 222.000 embarazos anuales (9). 
Tan sólo desde el punto de vista cuantitativo parece importante tratar de profundizar nuestros conocimientos sobre los problemas obstétricos de las adolescentes.

Pero el tema cobra mayor importancia aún, si se mira el impacto social y económico que puede tener la salud materno-infantil de las adolescentes.

Estas consideraciones, nos indujeron a estudiar una serie de características demográficas y gineco-obstétricas en las mujeres adolescentes que fueron atendidas en el Servicio de Obstetricia del Hospital Universitario de Caldas en el período comprendido entre el 10. de enero y el 31 de diciembre de 1989.

\section{Material y métodos}

Se aceptaron para el estudio 576 pacientes de 18 años o menos, que fueron atendidas en el Servicio de Obstetricia, entre el 1o. de enero y el 31 de diciembre de 1989.

Uno de los autores (MCM) revisó cuidadosamente las Historias Clínicas y consignó los datos de cada una de ellas en registros precodificados, específicamente diseñadas por él mismo, para Embarazo en Adolescentes. Dichos registros contienen 33 variables y 41 códigos diferentes. Para registrar morbimortalidad se empleó el sistema de codificación de la OMS (Novena Edición, 1975).

Los datos de estos registros fueron llevados al microcomputador y procesados allí, utilizando dBASE III+ Ver, 1.0, 1986 y Epiinfo versión 5.0, abril 1990.

Se hizo lo mismo con otro grupo de 544 pacientes primíparas con edades comprendidas entre 19 y 45 años atendidas en un período de seis meses, entre el 1-VII y el 31XII-89. Este grupo servirá como punto de comparación para investigar si las adolescentes tienen condiciones especiales que justifiquen planeación y manejo diferentes.

En este trabajo nos proponemos presentar las características del grupo de adolescentes y compararlas con "los estudios que hemos encontrado en la literatura nacional. En otro informe publicaremos los resultados de la comparación con el grupo de control. Dejamos abierta la posibilidad de hacer análisis detallados de subgrupos especiales, en ulteriores trabajos.

\section{Resultados}

\section{DISTRIBUCION POR EDADES}

\begin{tabular}{ccc}
\hline Edad & No. casos & \multicolumn{1}{c}{$\%$} \\
\hline 12 & 1 & 0.2 \\
13 & 2 & 0.3 \\
14 & 21 & 3.6 \\
15 & 50 & 8.7 \\
16 & 106 & 18.4 \\
17 & 175 & 30.4 \\
18 & 221 & 38.4 \\
\hline Total & $\mathbf{5 7 6}$ & $\mathbf{1 0 0 . 0}$ \\
\hline
\end{tabular}

Edad promedio: 16.9 años

Desviación estándar: 1.2
Con relación al número total de partos atendidos en el año (3.874), las adolescentes menores de 18 años represen$\tan$ el $14.9 \%$.

\section{DISTRIBUCION POR PESO}

\begin{tabular}{lcr}
\hline Peso & No. casos & \% \\
\hline $36-45$ & 10 & 2.5 \\
$46-55$ & 137 & 32.5 \\
$56-65$ & 189 & 44.9 \\
$66-75$ & 74 & 17.7 \\
$76-85$ & 10 & 2.2 \\
\hline Total & $\mathbf{4 2 0}$ & $\mathbf{1 0 0 . 0}$ \\
\hline
\end{tabular}

Peso promedio: $59.5 \mathrm{Kg}$.

Desviación estándar: 7.8

Subregistro: $34.2 \%$ (\% de registros sin dato)

\section{DISTRIBUCION POR TALLA}

\begin{tabular}{lcr}
\hline Talla & No. casos & $\%$ \\
\hline $135-139$ & 3 & 0.8 \\
$140-144$ & 17 & 4.8 \\
$145-149$ & 36 & 10.2 \\
$150-154$ & 110 & 31.0 \\
$155-159$ & 98 & 27.7 \\
$160-164$ & 66 & 18.6 \\
$165-169$ & 19 & 3.1 \\
$170-174$ & 4 & 1.1 \\
\hline Total & $\mathbf{3 5 3}$ & $\mathbf{1 0 0 . 0}$ \\
\hline
\end{tabular}

Talla promedio: $155.0 \mathrm{cms}$.

Desviación estándar: 6.3

Subregistro: 38.7

\section{DISTRIBUCION POR ESTADO CIVIL}

\begin{tabular}{lcc}
\hline Estado & No. Casos & \% \\
\hline Unión estable & 273 & 47.5 \\
Unión inestable & 302 & 52.5 \\
\hline Total & $\mathbf{5 7 5}$ & $\mathbf{1 0 0 . 0}$ \\
\hline
\end{tabular}

\section{DISTRIBUCION POR AÑOS DE ESTUDIO}

\begin{tabular}{lrr}
\hline \multicolumn{1}{c}{ Estudios } & No. Casos & \multicolumn{1}{c}{$\%$} \\
Analfabetas & 17 & 3.1 \\
Primaria incompleta & 154 & 27.7 \\
Primaria completa & 125 & 22.5 \\
Secundaria incompleta & 2450 & 44.1 \\
Secundaria completa & 12 & 2.2 \\
Superior incompleta & 3 & 0.5 \\
\hline Total & $\mathbf{5 5 6}$ & $\mathbf{1 0 0 . 0}$ \\
\hline Subregistro: & $3.5 \%$ &
\end{tabular}


6. DISTRIBUCION POR PROCEDENCIA

\begin{tabular}{lrr}
\hline Procedencia & No. Casos & \multicolumn{1}{c}{$\%$} \\
\hline Urbana & 476 & 82.6 \\
Rural & 100 & 17.4 \\
\hline Total & $\mathbf{5 7 6}$ & $\mathbf{1 0 0 . 0}$ \\
\hline
\end{tabular}

La edad promedio de la menarca fue de 12.8 años. Desviación standard: 1.3. El subregistro de este dato fue de $22.6 \%$.

En cuanto al número de embarazos el $85.1 \%$ de los adolescentes eran primigestantes. El $14.8 \%$ secundi o trigestantes.

\section{DISTRIBUCION POR No. CONTROLES PRE- NATALES}

\begin{tabular}{ccc}
\hline Controles & No. Casos & \% \\
\hline 0 & 116 & 20.4 \\
1 & 22 & 3.9 \\
2 & 51 & 8.9 \\
3 & 58 & 10.2 \\
4 & 88 & 15.4 \\
5 & 79 & 13.9 \\
6 & 58 & 10.2 \\
7 & 43 & 7.5 \\
8 & 55 & 9.6 \\
\hline Total & $\mathbf{5 7 0}$ & $\mathbf{1 0 0 . 0}$ \\
\hline
\end{tabular}

Promedio: 3.7

Desviación estándar: 2.6

El grupo etario de más bajo nivel de control prenatal fue el de 17 años, con $73.7 \%$.

\section{CONTROLES PRENATALES SEGUN INS- TRUCCION}

\begin{tabular}{|c|c|c|c|c|}
\hline Instrucción Control & No & $\%$ & $\mathrm{Si}$ & $\%$ \\
\hline Analfabetas & 6 & 35.3 & 11 & 64.7 \\
\hline Primaria incompleta & 45 & 29.2 & 109 & 70.8 \\
\hline Primaria completa & 25 & 20.0 & 100 & 80.0 \\
\hline Secundaria incompleta & 32 & 13.1 & 213 & 86.9 \\
\hline Secundaria completa & 3 & 25.0 & 9 & 75.0 \\
\hline Postsecundaria & 0 & 0.0 & 3 & 100.0 \\
\hline Total & 111 & & 445 & \\
\hline
\end{tabular}

Al comparar los controles prenatales de acuerdo con la procedencia urbana o rural de las pacientes no encontramos diferencia significativa.
9. MORBILIDAD EN EL EMBARAZO ACTUAL

\begin{tabular}{lcr}
\hline Patología & Frecuencia & \%* \\
\hline Ninguna & 347 & 60.2 \\
Infección urinaria & 89 & 15.5 \\
Tabaquismo & 41 & 7.1 \\
Amenaza parto pretérmino & 29 & 5.0 \\
Pre-eclampsia severa & 22 & 3.8 \\
Pre-eclampsia leve & 18 & 3.1 \\
Ruptura prematura de membranas & 17 & 2.9 \\
Condilomatosis & 14 & 2.4 \\
Incompatibilidad Rh & 10 & 1.7 \\
Sífilis (tratada) & 7 & 1.2 \\
Eclampsia & 5 & 0.9 \\
Embarazo gemelar & 5 & 0.9 \\
Amenaza de aborto & 3 & 0.5 \\
Otras & 32 & 5.5 \\
\hline
\end{tabular}

* Los porcentajes están calculados con respecto a 576 pacientes.

\section{DISTRIBUCION POR EDAD GESTACIONAL}

\begin{tabular}{ccc} 
Edad & No. Casos & \% \\
\hline $28-29$ & 2 & 0.3 \\
$30-31$ & 3 & 0.5 \\
$32-33$ & 5 & 0.9 \\
$34-35$ & 17 & 3.0 \\
$36-37$ & 49 & 8.5 \\
$38-39$ & 223 & 39.0 \\
$40-41$ & 254 & 44.4 \\
$42-43$ & 19 & 3.3 \\
\hline Total & 572 & 100.0 \\
\hline
\end{tabular}

Edad gestacional promedio: 39.0 semanas

Desviación estándar: 1.8

Subregistro: 0.7

La distribución de acuerdo con el tiempo de ruptura de membranas hasta el parto mostró $41.0 \%$ con intervalo de 16 horas. El $43.3 \%$ de las adolescentes tuvieron menos de 1 hora de ruptura de membranas.

La distribución según el tipo de presentación mostró cefálicas $92.7 \%$ (534 casos) pelvis $4.5 \%$ (26) compuesta $2.4 \%$ (14) y otras $0.4 \%$ (2).

La presentación fetal en relación con la edad, mostró una máxima incidencia de presentación de pelvis (7.5\%) en las pacientes de 16 años.

\section{DISTRIBUCIONSEGUNTERMINACION DEL PARTO}

\begin{tabular}{lcr}
\hline Parto & No. Casos & \multicolumn{1}{c}{$\%$} \\
\hline Normal & 444 & 77.1 \\
Con fórceps & 37 & 6.4 \\
Por cesárea & 91 & 15.8 \\
En pelvis (asistido) & 4 & 0.7 \\
\hline Total & $\mathbf{5 7 6}$ & $\mathbf{1 0 0 . 0}$ \\
\hline
\end{tabular}




\section{PARTO (EN PORCENTAJES) SEGUN TIPO DE PRESENTACION}

\begin{tabular}{lccrr} 
Presentación & Parto normal & Fórceps & Cesárea & Pelvis \\
\hline Cefálica & 80.3 & 6.9 & 12.7 & 0.0 \\
Frente & 0.0 & 0.0 & 100.0 & 0.0 \\
Pelvis & 0.0 & 0.0 & 80.8 & 19.2 \\
Hombro & 0.0 & 0.0 & 100.0 & 0.0 \\
Compuesta & 100.0 & 0.0 & 0.0 & 0.0 \\
\hline Total & $\mathbf{4 4 4}$ & $\mathbf{3 7}$ & $\mathbf{9 1}$ & $\mathbf{4}$ \\
\hline
\end{tabular}

13. INDICACIONES DE PARTOS INTERVENIDOS (CESAREA Y FORCEPS)

\begin{tabular}{lcc} 
Indicaciones & Frecuencia & $\% *$ \\
T. de parto disfuncional & 57 & 44.5 \\
Desproporción cefalopélvica & 26 & 20.3 \\
S.F.A. & 22 & 17.2 \\
Presentación pelvis & 21 & 16.4 \\
Inducción fallida & 16 & 12.5 \\
Cesáreas, sin aclarar causa & 14 & 10.9 \\
Fórceps, sin especificar indicac. & 6 & 4.7 \\
Otras & 19 & 14.8 \\
\hline
\end{tabular}

* Los porcentajes están calculados con respecto a 128 pacientes.

El tipo de anestesia empleado en 91 cesáreas fue: general en $26.4 \%$, peridural en $53.8 \%$ y raquídea en $12.1 \%$.

Las cesáreas fueron realizadas por médicos residentes de primero, segundo y tercer nivel, en $85.8 \%$ de los casos. El resto $(14.2 \%)$ por profesores e internos.

En los partos con fórceps se emplearon espátulas de Velasco (67.6\%), fórceps de Simpson (18.9\%), de Kielland $(8.1 \%)$ y de Elliot $(5.4 \%)$.

\section{DISTRIBUCION SEGUN TIPO DE EPISIOTOMIA}

\begin{tabular}{lcr}
\hline Episiotomía & No. Casos & \% \\
\hline Ninguna & 134 & 23.3 \\
Mediana & 398 & 69.1 \\
Mediolateral & 44 & 7.6 \\
\hline Total & $\mathbf{5 7 6}$ & $\mathbf{1 0 0 . 0}$
\end{tabular}

\section{DISTRIBUCION SEGUN DESGARROS PERINEALES}

\begin{tabular}{lcr}
\hline Desgarro & No. Casos & $\%$ \\
\hline Ninguno & 446 & 77.4 \\
Grado I & 13 & 2.3 \\
Grado II & 21 & 3.6 \\
Grado III & 50 & 8.7 \\
Grado IV & 42 & 7.3 \\
Otros desgarros & 4 & 0.7 \\
\hline Total & $\mathbf{5 7 6}$ & $\mathbf{1 0 0 . 0}$ \\
\hline
\end{tabular}

Al hacer la distribución de los desgarros perineales según el tipo de episiotomía, encontramos un número mayor de desgarros III y IV cuando se empleó la episiotomía mediana que cuando se hizo la mediolateral o cuando no se hizo episiotomía.

\section{COMPLICACIONES DEL PARTO}

\begin{tabular}{lcc}
\hline Patología & Frecuencia & \%* \\
\hline Ninguna & 428 & 74.3 \\
Atonía uterina & 50 & 8.7 \\
Parto pretérmino & 40 & 6.9 \\
T. parto disfuncional & 35 & 6.1 \\
S.F.A. & 34 & 5.9 \\
Corioamnionitis & 10 & 1.7 \\
Otras & 14 & 2.4 \\
\hline
\end{tabular}

* Los porcentajes están calculados con respecto a 576 pacientes.

\section{DISTRIBUCION SEGUN SEXO DEL RECIEN NACIDO}

\begin{tabular}{lcr}
\hline Sexo & No. Casos & \% \\
\hline Masculino & 312 & 54.2 \\
Femenino & 264 & 45.8 \\
\hline Total & $\mathbf{5 7 6}$ & $\mathbf{1 0 0 . 0}$ \\
\hline
\end{tabular}

18. DISTRIBUCION SEGUN PESO DEL RECIEN NACIDO

\begin{tabular}{ccc}
\hline Peso & No. Casos & \% \\
\hline $500-999$ & 1 & 0.2 \\
$1000-1499$ & 3 & 0.5 \\
$1500-1999$ & 9 & 1.6 \\
$2000-2499$ & 45 & 7.8 \\
$2500-2999$ & 165 & 28.8 \\
$3000-3499$ & 247 & 43.0 \\
$3500-3999$ & 92 & 16.0 \\
$4000-4499$ & 12 & 2.1 \\
\hline Total & $\mathbf{5 7 4}$ & $\mathbf{1 0 0 . 0}$ \\
\hline
\end{tabular}

Peso promedio del recién nacido: $3.047 \mathrm{~g}$. Desviación estándar: $479 \mathrm{~g}$.

\section{DISTRIBUCIONSEGUN TALLA DEL RECIEN} NACIDO

\begin{tabular}{ccc}
\hline Talla & No. Casos & \% \\
\hline 34 & 1 & 0.2 \\
36 & 1 & 0.2 \\
37 & 1 & 0.2 \\
39 & 2 & 0.3 \\
40 & 3 & 0.5 \\
41 & 4 & 0.7 \\
42 & 3 & 0.5
\end{tabular}




\begin{tabular}{ccc} 
Talla No. & Casos & \% \\
\hline 43 & 7 & 1.2 \\
44 & 8 & 1.4 \\
45 & 29 & 5.1 \\
46 & 44 & 7.7 \\
47 & 75 & 13.1 \\
48 & 105 & 18.3 \\
49 & 100 & 17.4 \\
50 & 116 & 20.2 \\
51 & 44 & 7.7 \\
52 & 28 & 4.9 \\
53 & 3 & 0.5 \\
\hline Total & $\mathbf{5 7 4}$ & $\mathbf{1 0 0 . 0}$
\end{tabular}

Promedio: $48 \mathrm{~cm}$

Desviación estándar: 2

\section{DISTRIBUCION SEGUN INDICE DE APGAR AL MINUTO}

\begin{tabular}{lcr}
\hline \multicolumn{1}{c}{ Apgar } & No. Casos & \multicolumn{1}{c}{$\%$} \\
\hline Mortinatos & 4 & 0.7 \\
$1-3$ & 11 & 2.0 \\
$4-6$ & 97 & 16.8 \\
7 ó más & 464 & 80.6 \\
\hline Total & $\mathbf{5 7 6}$ & $\mathbf{1 0 0 . 0}$ \\
\hline
\end{tabular}

La distribución del índice de Apgar al minuto, según el tipo de presentación, mostró 7 ó más en $82.0 \%$ de cefálicas, en $53.9 \%$ de pelvis y $78.7 \%$ compuestas.

El puntaje de Apgar al minuto según el tipo de parto mostró 7 ó más en $84.2 \%$ de los normales, en $50.0 \%$ de los partos en pelvis, en $54.0 \%$ de casos con fórceps y en $74.8 \%$ de partos por cesárea.

\section{DISTRIBUCION SEGUN INDICE DE APGAR A LOS CINCO MINUTOS}

\begin{tabular}{lcc}
\hline Apgar & No. Casos & \% \\
\hline Mortinatos & 4 & 0.7 \\
$1-3$ & 1 & 0.2 \\
$4-6$ & 16 & 2.8 \\
7 ó más & 555 & 96.3 \\
\hline Total & $\mathbf{5 7 6}$ & $\mathbf{1 0 0 . 0}$
\end{tabular}

De acuerdo con el tipo de presentación, el índice de Apgar a los 5 minutos fue de 7 ó más en $96.6 \%$ de cefálicas y en $88.4 \%$ de pelvis.

Según el tipo de parto, el índice de Apgar a los 5 minutos fue de 7 ó más en $96.2 \%$ de partos normales.

\section{MORBILIDAD NEONATAL}

\begin{tabular}{lcr}
\hline Patología & Frecuencia & \%* \\
\hline Ninguna & 328 & 57.3 \\
Circular cordón & 83 & 14.5 \\
S.F.A. & 52 & 9.1 \\
R.N. Pretérmino & 41 & 7.2 \\
Bajo peso/Edad gestacional & 33 & 5.8 \\
Caput & 29 & 5.1 \\
Alteraciones congénitas & 22 & 3.8 \\
R.C.I.U. & 11 & 1.9 \\
R.N. macrosómico & 10 & 1.7 \\
S.D.R. & 8 & 1.4 \\
Síndrome aspiración meconio & 8 & 1.4 \\
Conjuntivitis purulenta & 7 & 1.2 \\
Otras & 20 & 3.5 \\
\hline
\end{tabular}

* Los porcentajes están calculados con respecto a 577 neonatos vivos.

\section{COMPLICACIONES DEL PUERPERIO}

\begin{tabular}{lcr}
\hline Patología & Frecuencia & $\%^{*}$ \\
Ninguna & 481 & 83.5 \\
Anemia 11 g\% & 86 & 15.0 \\
Endometritis puerperal & 11 & 1.9 \\
Infección herida quirúrgica & 6 & 1.0 \\
Otras & 8 & 1.4 \\
\hline
\end{tabular}

* Los porcentajes están calculados con respecto a 576 pacientes.

\section{Discusión}

En resumen, se trata de un grupo de mujeres adolescentes, cuyas características podemos sintetizar así:

\section{CARACTERISTICAS DE LAS ADOLESCENTES}

\begin{tabular}{|c|c|c|c|}
\hline Características & $\%$ & Promedio De & Desv. Estánd. \\
\hline Edad & & 16.9 años & 1.2 \\
\hline Peso & & $59.5 \mathrm{Kg}$. & 7.8 \\
\hline Talla & & $155 \mathrm{~cm}$. & 6.3 \\
\hline Unión inestable & $52.5 \%$ & & \\
\hline Procedencia urbana & $82.6 \%$ & & \\
\hline \multicolumn{4}{|l|}{$\begin{array}{l}\text { Educación primaria } \\
\text { completa }\end{array}$} \\
\hline o incompleta & $84.1 \%$ & & \\
\hline Primigestantes & $88.5 \%$ & & \\
\hline Con embarazo a término & $86.4 \%$ & & \\
\hline Menarca & & 12.8 años & 1.3 \\
\hline Edad gestacional & & 39 semanas & 1.8 \\
\hline Presentación: cefálica & $92.7 \%$ & & \\
\hline Parto normal & $77.1 \%$ & & \\
\hline Episiotomía: mediana & $69.1 \%$ & & \\
\hline \multicolumn{4}{|c|}{ Recién nacido: varones $54.2 \%$} \\
\hline Peso del R.N. & & $3047 \mathrm{~g}$. & 477 \\
\hline Talla del R.N. & & $48 \mathrm{~cm}$. & 2 \\
\hline Apgar al minuto: 7 ó más & $80.6 \%$ & & \\
\hline \multicolumn{4}{|c|}{ Apgar a los 5 minutos: } \\
\hline 7 ó más & $96.3 \%$ & & \\
\hline
\end{tabular}

La morbilidad más importante en el grupo estudiado fue preeclampsia-eclampsia, infección urinaria, tabaquismo, amenaza de parto pretérmino, ruptura prematura de membranas, condilomatosis e incompatibilidad Rh. (Tabla 9). 
Hubo 128 partos intervenidos: 37 con fórceps y 91 por cesárea. Las indicaciones de estos procedimientos se presentan en la Tabla 13.

La falta promedio de nuestras adolescentes fue de 155 $\mathrm{cm}$. más o menos 6 .

$\mathrm{El}$ analfabetismo es bajo: $3.1 \%$. El analfabetismo funcional (tres años o menos de estudio), fue de $19.6 \%$. Esto nos da alguna idea sobre el nivel cultural del grupo estudiado.

Econtramos unión estable en el $47.5 \%$.

Los datos sobre procedencia de las pacientes nos indican que la acción del Hospital se ejerce principalmente sobre población urbana: $82.6 \%$.

Las adolescentes embarazadas que acuden al Hospital Universitario, son en su inmensa mayoría primigestantes: $85.1 \%$.

Se observa una relación inversa entre la edad y el control prenatal, aunque el escaso número de adolescentes menores de 15 años no permite sacar conclusiones seguras al respecto. Por el contrario, la relación es directa con el nivel de instrucción, en términos generales.

Nos llama la atención el porcentaje bajo de preeclampsia y eclampsia: $7.8 \%$.

Las presentaciones de pelvis ocurrieron mas frecuentemente en el grupo de 16 años: $7.5 \%$.

Es muy importante el porcentaje de partos intervenidos y en pelvis (que consideramos anormal): $22.9 \%$. Dentro de los patrones actuales llama la atención, como bajo, el porcentaje de cesáreas: $15.8 \%$.

Hay que señalar el hecho de que el $80.8 \%$ de los partos en presentación de pelvis fueron por cesárea.

La episiotomía más utilizada (69.1\%) fue la mediana. Llama la atención que los desgarros III y IV se producen precisamente con este tipo de episiotomía. En la práctica real del Hospital Universitario, la episiotomía mediana no previene este tipo de desgarros. Por el contrario, parece favorecerlos.

El resultado inmediato del parto, a juzgar por el índice de Apgar nos muestra un $16.8 \%$ de recién nacidos moderadamente deprimidos al minuto y sólo $2.8 \%$ a los cinco minutos, lo cual parece satisfactorio.

Analizando el índice de Apgar en relación con la presentación se observa que la depresión moderada del recién nacido al minuto ocurre en $38.4 \%$ de los partos en presentación de pelvis y de $50.0 \%$ a los cinco minutos. Aún cuando el parto en presentación de pelvis se realice por cesárea, la depresión moderada del recién nacido sigue siendo de $22.0 \%$ y la severa de 3.3 al minuto.

En cuanto a las complicaciones del puerperio, no disponemos de un registro satisfactorio, ya que las pacientes permanecen hospitalizadas en promedio 2.8 días, período muy breve para que se manifiesten muchas de ellas.

Promedio de días de hospitalización de las pacientes: 2.9 días

Desviación estándar: 3.3 días

Rango: 49 días.

\section{COMPARACION CON ALGUNOS ESTU- DIOS PREVIOS NACIONALES}

Vamos a resumir en el cuadro siguiente, aquellos aspectos que hemos encontrado susceptibles de comparación con algunos trabajos previos.

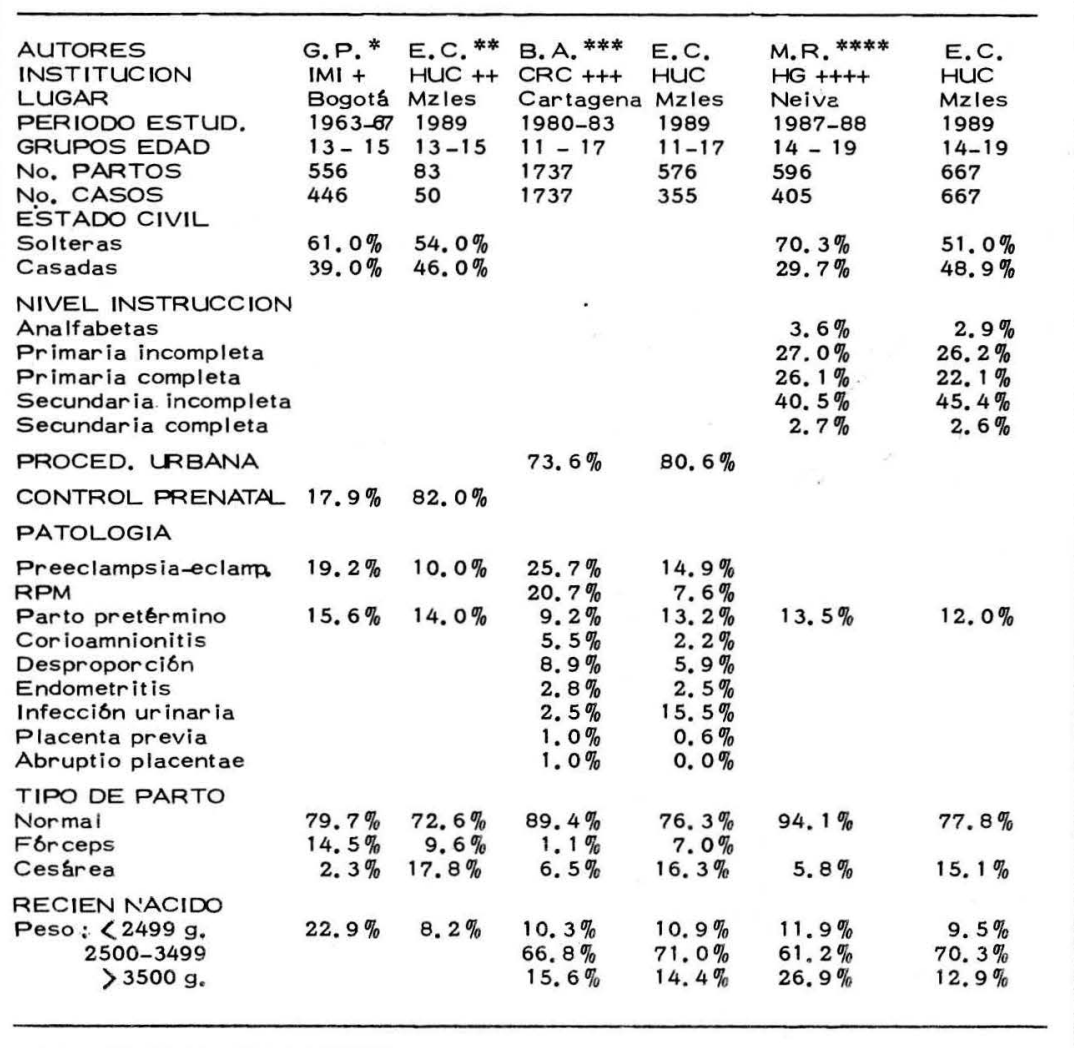

\footnotetext{
* gomez palacino + Instituto Materno Infantil

** ESPARZA - CALLE ++ Hospital Universitario de Clds. *** BARRIOS AMAYA Y COLS. +++ Clínica Rafael Calvo **** MORENO ROJAS ++++ Hospital General
} 
En el cuadro se presentan algunos datos comparables con los nuestros, de los estudios de Gómez Palacino, Barrios Amaya, y Moreno Rojas.

Los grupos de edad corresponden a los que cada autor estudia específicamente.

El número de partos se refiere a esos mismos grupos etáreos.

El número de casos alude a los subgrupos COMPARABLES.

Sobre el estudio de Gómez Palacino debemos aclarar que $\operatorname{los} 446$ casos corresponden a pacientes de 15 años. Sus datos sobre estado civil, control prenatal, preeclampsiaeclampsia y parto pretérmino, se refieren únicamente a este subgrupo. Los de tipo de parto y peso del recién nacido, corresponden a todo el grupo de 13 a 15 años (556 casos).

A los datos nuestros se aplican análogas consideraciones, (83 casos de 13 a 15 años; 50 correspondientes a mujeres de 15 años).

Vale la pena destacar la notable diferencia en porcentaje de pacientes con control prenatal y preeclampsiaeclampsia, en el grupo de 15 años, con resultados mucho mejores en nuestras pacientes. Igualmente significativas clínicamente, nos parecen las diferencias en cuanto a porcentajes de cesáreas y de bajo peso al nacer.

Debemos señalar nuestras diferencias más notables en el estudio de Barrios Amaya y col.: incidencia de pre- eclampsia-eclampsia, ruptura de membranas, corioamnionitis, infección urinaria, uso de fórceps y cesárea.

Con el estudio de Moreno Rojas, hay que subrayar las diferencias clínicamente importantes en cuanto a estado civil de las pacientes, partos normales, cesáreas y pesos de los recién nacidos superiores a $3500 \mathrm{~g}$.

Los demás estudios consultados no nos permitieron por su diseño o por su contenido, establecer comparación alguna con el nuestro.

\section{Conclusiones}

Es prácticamente imposible realizar comparaciones entre los estudios publicados en la literatura, por la gran heterogeneidad de los mismos.

Sigue siendo deseable la realización de un estudio prospectivo cooperativo con el fin de poder dilucidar con mejor probabilidad de acertar, problemas relacionados con el embarazo y parto en adolescentes.

Hemos presentado en este trabajo las características del grupo estudiado, las cuales quedaron resumidas en la Tabla 24.

En un trabajo próximo compararemos el grupo de adolescentes primigestantes con otro de primigestantes de 19 a 30 años, con el propósito de ver si hay diferencias y si éstas son o no significativas.

\section{BIBLIOGRAFIA}

1. Barrios JA. y Cols. Aspectos perinatales de las adolescentes. Rev. Col. Obstet. Ginecol. 1985; 36: 102.

2. Duarte Contreras A. Embarazo y prostitución en adolescentes. Rev. Col. Obstet. Ginecol. 1979; 30: 1.

3. Duarte Contreras A. y Barreto López A. Factores determinantes del embarazo en adolescentes solteras. Rev. Col. Obstet. Ginecol. 1985; 36: 291.

4. Duarte Contreras A. Manejo de la adolescente embarazada. Aspectos médicos. Rev. Col. Obstet. Ginecol. 1900; 41: 25.

5. Duarte Contreras A. Rev. Col. Obstet. Ginecol. 1973; 14: 259.

6. Gómez Palacino JA. Obstetricia en adolescentes. Rev. Col. Obstet. Ginecol. 1972; 23: 353.

7. Jaramillo UR. Adolescencia. Rev. Col. Obstet. Ginecol. 1984; 35: 401.
8. Moreno Rojas A. Adolescentes y embarazo. Hospital General de Neiva. 1988. Rev. Col. Obstet. Ginecol. 1989; 40: 227.

9. Remez L. Fecundidad en adolescentes en América Latina y el Caribe: Examen del problema. Persp. Int. en Plan Fam. Número Especial 1990; 24-28.

10. Rizo GA. y Prada SE. La fecundidad de la población de 10-19 años en Colombia. Rev. Col. Obstet. Ginecol. 1982; 33: 357.

11. Tovar MC. y Cols. Estudio comparativo de embarazadas adolescentes versus embarazadas adultas jóvenes en el Centro Hospital Primitivo Iglesias de la ciudad de Cali. Rev. Col. Obstet. Ginecol. 1987; 38 436.

12. Uriza Gutiérrez G. Embarazo en adolescentes. Rev. Col. Obstet. Ginecol. 1983; 34: 102. 